\title{
Etiology and pathogenesis of Parkinson's disease: role of mitochondrial pathology
}

\author{
This article was published in the following Dove Press journal: \\ Research and Reports in Biochemistry \\ 15 May 2013 \\ Number of times this article has been viewed
}

\section{Denis Ottolini \\ Tito Calì \\ Marisa Brini}

Department of Comparative Biomedicine and Food Science, University of Padova, Padova, Italy
Correspondence: Marisa Brini Department of Comparative Biomedicine and Food Science, University of Padova, 3 Viale G Colombo, Padova 35131, Italy Tel +39049827 6I50

Fax +39049827 6I 25

Email marisa.brini@unipd.it

\begin{abstract}
Neurons critically rely on mitochondrial activity: they are characterized by high energy demand and they are totally dependent on the process of oxidative phosphorylation to produce adenosine triphosphate. Thus, any impairment in mitochondrial function results in neuronal damage and degeneration. Some particular neuronal populations are more susceptible to mitochondrial damage, as it has been recently proposed for the ventral midbrain dopaminergic neurons, the degeneration of which represents a clinical sign of Parkinson's disease. Different cellular pathways are involved in the pathogenesis of this neurodegenerative disease, but intriguingly both sporadic and familial forms share common features that essentially recapitulate mitochondrial dysfunction. Mitochondrial biogenesis, bioenergetics, mitochondria dynamics, and quality-control process are the main affected pathways. General consensus agrees on the possibility that deficiency in these processes may represent the cause rather than the consequence of neurodegeneration. In this review, we will discuss these aspects and the substantial achievements that have been reached in recent years in identifying specific defects in precise biological processes, eg, mitochondrial quality control. The development of cell and animal genetic models has been an important tool to dissect numerous molecular details; for this reason, we will mainly refer to experiments performed on them.
\end{abstract}

Keywords: mitochondria, Parkinson's disease, $\alpha$-synuclein, PINK1/parkin, DJ-1, LRRK2

\section{Introduction}

Parkinson's disease (PD) is a progressive neurodegenerative movement disorder affecting approximately $2 \%$ of the population over 65 years of age. It is characterized by resting tremor, bradykinesia, rigidity, and postural instability, which are essentially caused by a decrease of dopamine levels in the brain. The main pathological hallmarks of PD are the loss of pigmentation in the substantia nigra pars compacta (due to the loss of neuromelanin-containing dopaminergic neurons) and the presence of cytoplasmic inclusions, commonly referred to as Lewy bodies, of which $\alpha$-synuclein ( $\alpha$-syn) is the principal component. ${ }^{1}$

PD occurs mostly sporadically, and its association with complex I deficiency has been established since it was clearly documented that 1-methyl-4-phenyl-1,2,3,6-tetrahydropyridine (MPTP), an analogue of the narcotic meperidine, caused Parkinsonism in humans. ${ }^{2}$ The MPTP neurotoxic active metabolite 1-methyl-4-phenylpyridinium $\left(\mathrm{MPP}^{+}\right)$accumulates in the mitochondrial matrix and inhibits the oxidative phosphorylation by reducing the activity of complex $\mathrm{I}^{2}$ Chronic systemic administration of rotenone or other pesticides in rats results in neuropathological and behavioral changes resembling human phenotypes. ${ }^{3}$ In addition to complex I deficiency, oxidative stress 
has been causally linked to PD pathogenesis, and considering that the mitochondrial electron-transport chain (ETC) is the main site of reactive oxygen species (ROS) production, mitochondrial activity turns out to be once again a key determinant in balancing physiological or detrimental ROS production.

Since then, many efforts have been made to identify mitochondrial respiratory defects in PD patients, and over the last few decades cumulating evidence proved that association between mitochondrial dysfunction and PD is not only confined to complex I impairment., ${ }^{4,5}$

The identification of rare specific gene mutations that cause PD has reinforced the relevance of mitochondrial dysfunction in the etiology of the disease, but importantly it has also suggested a causative pathogenic role for it. Comprehensive description of the loci associated with
PD can be found elsewhere. ${ }^{6,7}$ In this review, we want to stress that all the so-far-identified PD-linked mutations affected mitochondrial-resident or mitochondrial-related gene products that in some circumstances may also localize to mitochondria. ${ }^{5}$ In particular, we will focus on five proteins conclusively linked to PD: phosphatase and tensin homolog-induced putative kinase 1 (PINK1), parkin, DJ-1, $\alpha$-synuclein ( $\alpha$-syn), and leucine-rich repeat kinase 2 (LRRK2). We will discuss recent progress in identifying their biological function and the consequences of their pathogenic mutations in genetic animal models, highlighting more compelling evidence for their interplay in the control of mitochondria function. Figure 1 summarizes several metabolic pathways that will be considered, ie, adenosine triphosphate (ATP) and ROS production, mitochondrial DNA (mtDNA) integrity, $\mathrm{Ca}^{2+}$ homeostasis,

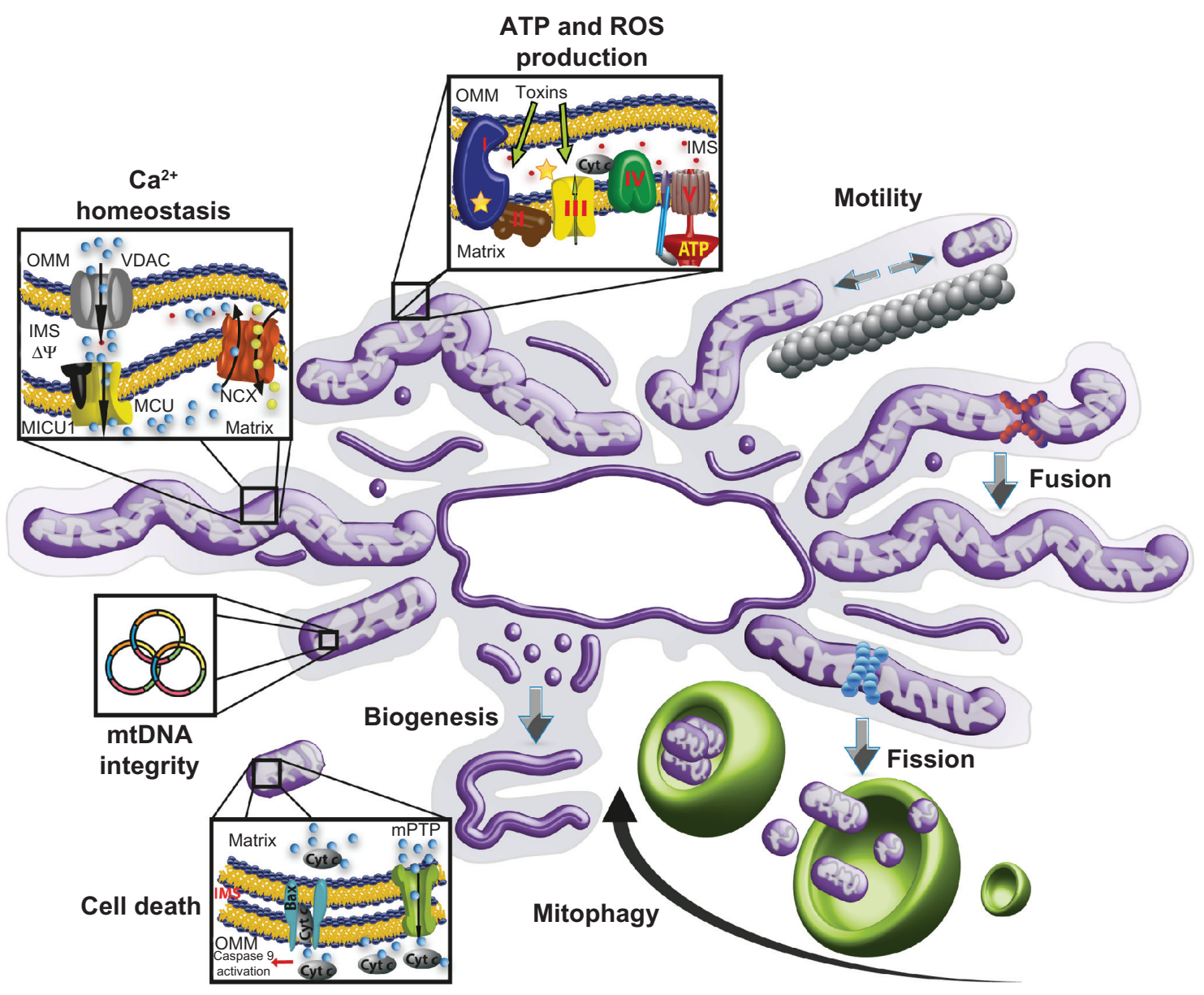

Figure I Mitochondria-related metabolic pathways that become compromised in Parkinson's disease.

Notes: Impairments in mitochondrial homeostasis maintenance occur at several checkpoints. Environmental toxins as well as mutations in Parkinson's disease (PD)-related proteins impact adenosine triphosphate (ATP) production and reactive oxygen species (ROS) scavenging. The process of mitochondria dynamics (trafficking, fission-fusion balance, and clearance by mitophagy) is under the control of proteins whose mutations are involved in the PD pathogenesis. PD-related proteins also participate, directly or indirectly, in mitochondrial biogenesis, maintenance of mitochondrial $\mathrm{Ca}^{2+}$ homeostasis and mitochondrial DNA (mtDNA) integrity, and lastly in mitochondria-related apoptosis.

Abbreviations: OMM, outer mitochondrial membrane; VDAC, voltage-dependent anion channel; IMS, intermembrane space; MICUI, mitochondrial Ca ${ }^{2+}$ uptake I; MCU, mitochondrial $\mathrm{Ca}^{2+}$ uniporter; NCX, sodium-calcium exchanger; mPTP, mitochondrial permeability transition pore; cyt c, cytochrome c. 
and the processes of mitochondria dynamics and quality control.

\section{Familial PD-related proteins: a brief overview}

The PD-causing gene products are not mitochondrial proteins per se, except for the serine/threonine kinase PINK1. However, there is extensive (although often contradictory) literature showing that under certain circumstances, all of them may associate with mitochondria.

PINK1 is synthesized as a $66 \mathrm{kDa}$ precursor protein that bears an atypical mitochondrial targeting sequence, a transmembrane domain spanning the mitochondrial membranes, and a kinase domain that faces the cytosol. The processing, stability and subcellular distribution of PINK1 are dynamically regulated. Several intermediate maturation products have been described (see below), albeit their specific function, if any, is not evident. Initial reports suggested that PINK1 had a protective role against stress-induced cell death, since its depletion rendered PINK1-deficient cells more susceptible to apoptosis induced by mitochondrial toxins. ${ }^{8,9}$ Although the putative targets of PINK1 activity remain elusive, the phosphorylation of the mitochondrial molecular chaperone heatshock protein of $75 \mathrm{kDa}$, also known as tumor necrosis factor receptor-associated protein 1 , resulted in increased neuronal survival against oxidative stress or heat shock through the blockage of cytochrome $c$ release from mitochondria. ${ }^{10}$ PINK1 knockout mice showed reduction mainly in complex I activity but also in complexes II and III. ${ }^{11,12}$ In the last few years, the role of PINK1 in the regulation of mitochondria fission/ fusion and in the process of mitophagy, an organelle-selective autophagy, ${ }^{13,14}$ has increasingly emerged. ${ }^{15-18}$ This action, which turned out to be relevant for the pathogenesis of PD and other neurodegenerative conditions as well, will be discussed in detail in following paragraphs.

Parkin is a multifunctional E3 ubiquitin ligase that mediates ubiquitination of several target proteins, including itself. Parkin knockout mice exhibited normal brain morphology, but increased striatal extracellular dopamine levels and reduced levels of mitochondrial proteins involved in oxidative phosphorylation. ${ }^{19}$ Conversely, parkin overexpression prevents mitochondrial swelling and stress-induced apoptosis in cultured cells. ${ }^{20-22}$ Parkin is primarily cytosolic, but it is selectively recruited to damaged or uncoupled mitochondria to mediate their terminal clearance by mitophagy. ${ }^{23}$ This recruitment is mediated by PINK1 accumulation at the outer mitochondrial membrane (OMM) and requires its kinase activity. ${ }^{24}$ Following these observations, the initial idea that suggested an exclusive role for parkin in the targeting of dysfunctional proteins for proteasome-mediated degradation has been replaced by the proven hypothesis of its involvement in the removal of dysfunctional mitochondria through a PINK1-mediated pathway.

Like parkin, DJ-1 is predominantly cytosolic, but a fraction of the protein has been found in mitochondria after subcellular fractionation of transfected cells ${ }^{25-28}$ and mouse brain tissue. ${ }^{29}$ DJ-1 is a member of a large protein family and has homologues in nearly all organisms. The current view is that DJ-1 is a multifunctional protein that defends cells against ROS and mitochondrial damage during oxidative stress, although the details of its biochemical function remain unclear. A conserved cysteine residue (Cys106) is functionally essential: its oxidative modification has been proposed to allow DJ-1 to act as a sensor of cellular redox homeostasis, ${ }^{30}$ but also to act as antioxidant. ${ }^{31,32} \mathrm{~A}$ role for DJ-1 in modulating gene expression under oxidative conditions has been suggested. ${ }^{33}$

$\alpha$-Syn and LRRK2, two essentially cytosolic proteins involved in autosomal dominant forms of $\mathrm{PD}$, may be recruited to the mitochondrion under appropriate conditions. $\alpha$-Syn is a small protein implicated in maintaining the integrity of the synapse, in learning, and neuronal plasticity. ${ }^{34,35}$ In the brain, $\alpha$-syn is predominantly cytosolic, but a significant disruption of the mitochondrial membrane phospholipid composition was found in knockout mice together with the impairment of the complexes II and III of the respiratory chain. Recently, it has been proposed that $\alpha$-syn may regulate mitochondria dynamics by interfering with the fusion/fission machinery. ${ }^{36}$ (see below)

As suggested for other PD-related proteins, translocation of $\alpha$-syn from the cytosol to the OMM can occur, and appears to be dynamically regulated in response to oxidative or metabolic stress. However, it is unclear to what extent it represents a protective mechanism rather than a pathological process. ${ }^{37}$

LRRK2 is a large multidomain protein that belongs to a family known as ROCO, the members of which have a Ras of complex proteins (ROC) guanosine triphosphatase (GTPase) domain followed by a domain of unknown function (C-terminal of ROC, COR). LRRK2, similarly to other ROCO proteins, also possesses a kinase domain. ${ }^{38}$ The kinase activity is regulated by the GTPase activity, and in turn the ROC domain can be phosphorylated by the kinase domain. The reciprocal regulation of both these activities appears to be important to LRRK2 function. ${ }^{39}$ Interestingly, the majority of LRRK2 mutants have abnormally high kinase activity. ${ }^{40}$ Similarly to $\alpha$-syn, LRRK2 was found to be associated with 
the OMM in mitochondria-enriched fractions of transfected cells. LRRK2 immunoreactivity was also reported in the mitoplast fraction of mouse brain tissue. ${ }^{42}$

\section{Mitochondria and metabolism}

Mitochondria are commonly defined as the cellular "power plant." By oxidative phosphorylation, they produce the large majority of cellular ATP. Five multiprotein complexes (from I to $\mathrm{V}$ ) and two mobile electron carriers (ubiquinone and cytochrome $c$ ), localized in the inner mitochondrial membrane (IMM) and in the intermembrane space (IMS), deal with this important function. The ETC transfers electrons from reduced nicotinamide adenine dinucleotide (NADH) and flavin adenine dinucleotide $\left(\mathrm{FADH}_{2}\right)$ to oxygen and generates reducing potential that is employed by complexes I, III and IV to pump protons in the IMS. The gradient of protons will be dissipated by complex V (or ATP-synthase) to produce ATP according to the chemiosmotic theory. ${ }^{43}$ Neurons are particularly sensitive to changes in mitochondrial activity, since their numerous activities, ie, synaptic transmission, axonal/dendritic transport, and ion-pump activity, are highenergy-demanding processes. Thus, any impairment in mitochondrial physiology, even if subtle, can be detrimental for the wellness of the cells.

\section{Complex I deficits}

As already mentioned, complex I deficiency represents a common feature in PD pathogenesis: patients brain and cellular or animal PD models display reduced complex I activity, increased ROS generation and as a consequence deficiency in ATP production. For years, the peculiar vulnerability of dopaminergic neurons of substantia nigra has been attributed to these defects, further exacerbated by the high oxidative burden and low antioxidant capacity that characterize this specific neuronal population.

Following the identification of nuclear genes whose mutations are associated with PD, numerous studies have systematically searched for a relationship between the function of their products and defects in complex I activity to link idiopathic and genetic forms of the disease. Thus, first investigation has analyzed the effect of such proteins on the mitochondrial respiratory complexes and on ATP production. Several reports have appeared describing the direct interaction of some PD-related proteins and complex I subunits, proposing either a direct role in the assembly, in the formation of higher-order complexes, or in the regulation of the enzymatic activity.

It has been shown that the silencing of PINK1 gene impairs activity of the complex I (and to a lesser extent complexes
II and III) thus compromising the respiratory capacity of mitochondria. ${ }^{9,11,44}$ No differences were instead observed in the expression levels of the subunits of the respiratory chain complexes. Recently, a novel regulatory role of PINK1 in the maintenance of complex IV activity has also been proposed. ${ }^{45}$ A direct link between PINK1 and complex I activity has been established by experiments showing that the reintroduction of human wild-type (wt) PINK1, but not of its disease-associated mutants, rescues complex I deficiency in fruit fly and mouse models lacking PINK 1. ${ }^{12}$ However, the precise mechanism of this action is still unclear. Interestingly, it has been shown that overexpression of the mammalian complex I homologue yeast NADH dehydrogenase, but not the modulation of mitochondrial fission and fusion, rescues complex I deficiency and mitochondrial pathology observed in PINK1 mutant flies, suggesting that alteration of mitochondrial structure is secondary to complex I deficiency. ${ }^{46}$ Differently to PINK1, parkin depletion has been shown to induce consistent reductions in several subunits of complexes I and IV and to reduce respiratory capacity of isolated mitochondria. ${ }^{19}$ Consistently, deficiency in complex I activity has also been reported in parkin-mutant fibroblast from PD patients. ${ }^{47,48}$

As for DJ-1, it has been shown to colocalize with complex I and maintain its activity by direct interaction with two nuclear and mitochondrial DNA-encoded complex I subunits, NDUFA4 and ND1, respectively. ${ }^{49}$ Accordingly, DJ-1-knockdown cells displayed reduced complex I activity. ${ }^{50}$ Interestingly, DJ-1 overexpression protected astrocytes against rotenone-induced toxicity, while its knockdown increased the neurotoxicity of pesticides that specifically inhibit complex I but not of inhibitors of complexes II-V, which primarily induce oxidative stress. ${ }^{51}$ Recently, a mechanism for this action has been proposed: DJ-1 may have a direct role in controlling the assembly of complex I by affecting the levels of NDUFS1, a nuclearencoded mitochondrial subunit. ${ }^{52}$ This finding suggests that the mitochondrial impairment resulting from DJ-1 loss of function cannot be exclusively attributed to the loss of its antioxidant role.

Endogenous levels of $\alpha$-syn are required to maintain complexes I and III activity and guarantee ETC operation. ${ }^{53}$ On the other side, its overexpression or accumulation has also been associated with impairment of complex I activity, ${ }^{54-56}$ suggesting that it may have the opposite effect depending on its expression levels and its monomeric/oligomeric or aggregation state ${ }^{57}$ (see Figure 2). Immunoprecipitation assay and yeast two-hybrid screening revealed physical $\alpha$-syn association with complexes I and IV. ${ }^{54,58}$ In vitro incubation 
of isolated rat brain mitochondria with recombinant human $\alpha$-syn leads to dose-dependent loss of mitochondrial transmembrane potential and impaired oxidative phosphorylation, possibly due to interference with the lipid composition of mitochondrial membranes, rather than with the activities of the respiratory chain complexes. ${ }^{59}$ In any case, independently of the mechanism of action, $\alpha$-syn accumulation resulted in complex I inhibition, reduced mitochondrial membrane potential, and ATP production. Accordingly, transgenic mice overexpressing mutant $\alpha$-syn display altered mitochondrial function and structure. ${ }^{60}$

No direct link between deficits in complex I and LRRK2 mutations has been established. ${ }^{61,62}$ LRRK2 knockout mice did not display either increased or decreased susceptibility to MPTP toxin, suggesting that it is not implicated in regulating complex I activity. ${ }^{63}$ However, cultured dopaminergic cells from brain of mice expressing mutant LRRK2 and fibroblast from PD patients display mitochondrial pathology, decreased ATP production, and reduced mitochondrial membrane potential. ${ }^{61,62}$

\section{Oxidative stress}

Compared to glycolysis, in which only two molecules of ATP are produced, the oxidative phosphorylation produces 36 molecules of ATP from one single molecule of glucose. The price of this advantageous ATP production is the generation of ROS. While the ETC is working, about $5 \%$ of electrons leak from complexes I and III and cause partial reduction of $\mathrm{O}_{2}$, creating the toxic, stable, and extremely reactive superoxide anion $\mathrm{O}_{2}{ }^{--}$. Mitochondria contain ROS-detoxifying enzymes, eg, manganese superoxide dismutase, which transforms $\mathrm{O}_{2}{ }^{--}$into $\mathrm{H}_{2} \mathrm{O}_{2}$ that in turn is converted to $\mathrm{O}_{2}$ and $\mathrm{H}_{2} \mathrm{O}$ by glutathione peroxidase, and other nonenzymatic ROS-scavenging components (as glutathione, coenzyme $\mathrm{Q}_{10}$, cytochrome $c$ ) that contribute to the antioxidant defense system. In the case of ETC dysfunction, ROS production increases, thus augmenting the risk of oxidative damage to mtDNA, proteins, or lipids and further promoting oxidative stress, metabolic dysfunction, and even apoptotic cell death. As aforementioned, this risk is particularly high for neuronal cells, and in particular for dopaminergic neurons. In fact, the dopamine metabolism and the peculiarly low levels of antioxidants could further increase ROS generation, making them particularly exposed to oxidative stress damage. ${ }^{64} \mathrm{~A}$ causative link between ROS and PD has been supported by a number of studies performed in model cells and investigating the biological function of the PD-related proteins. In particular, the role of DJ-1 against oxidative stress has been investigated, since it has been characterized as a peroxiredoxin-like peroxidase. ${ }^{30}$ It counteracts oxidative stress through direct oxidation of its reactive cysteine residue 106 to sulfinic (and to sulfenic and

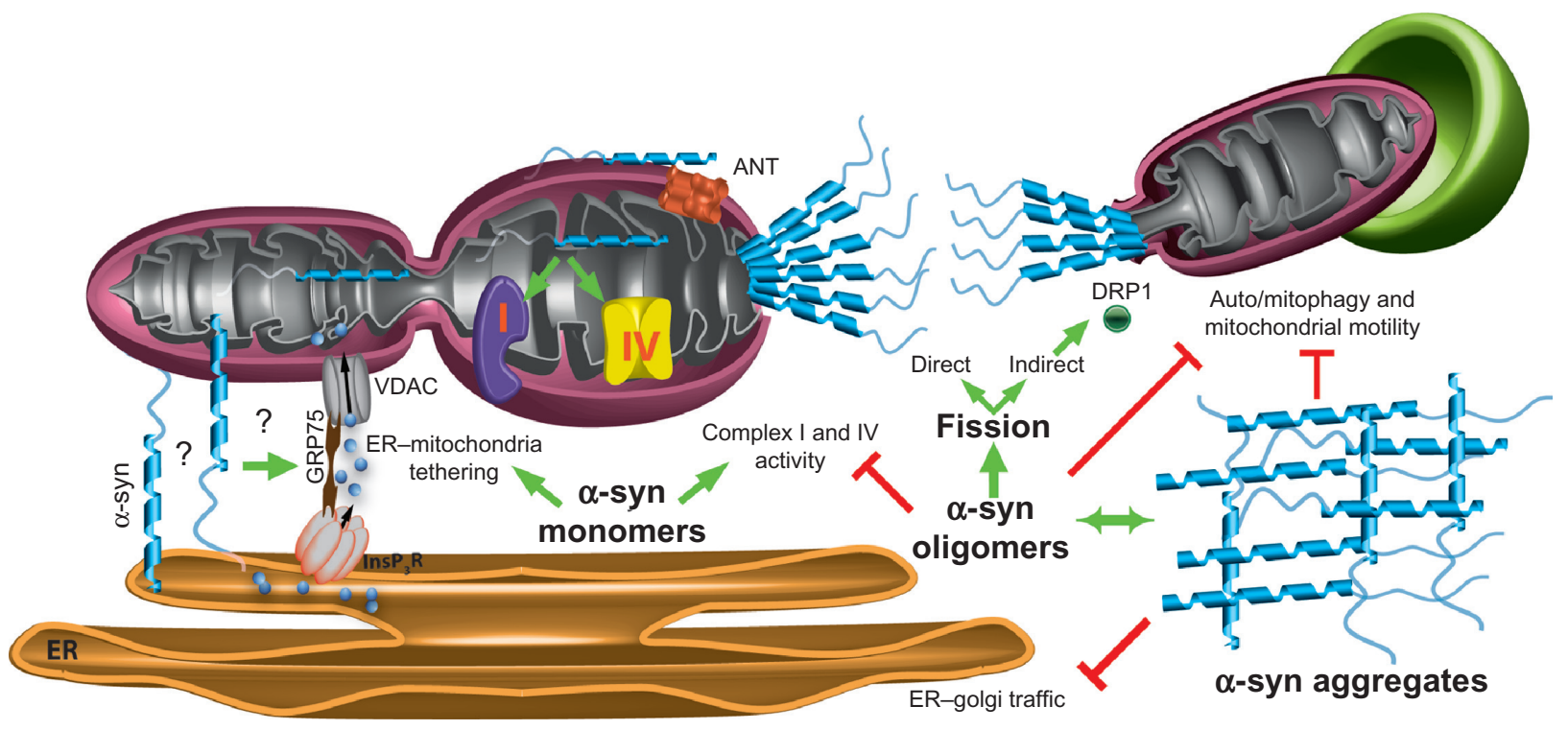

Figure 2 Physiological and pathological functions of $\alpha$-synuclein ( $\alpha$-syn) on mitochondria.

Notes: $\alpha$-Syn loss of function (induced both by its exaggerated overexpression and by its silencing) affects complex I activity, mitochondrial membrane integrity, ER-Golgi vesicles trafficking, and ER-mitochondria communication, thus impairing mitochondrial function. Red bar-headed lines and green arrow-headed lines indicate inhibitory and stimulatory effects, respectively. The effect, in some cases, depends on the $\alpha$-syn aggregation state, as indicated. The exact orientation of $\alpha$-syn at the ER-mitochondria contact sites as well as whether it plays a direct or an indirect role in their formation is still unknown (?).

Abbreviations: ER, endoplasmic reticulum; VDAC, voltage-dependent anion channel; ANT, adenine-nucleotide translocase; Grp75, glucose-regulated protein 75; DRPI, dynamin-related protein I. 
sulfonic in case of high oxidant burn) acid, thus guaranteeing neuroprotection. ${ }^{32}$ Cultured neurons from mouse models, fibroblasts, and lymphoblasts from patients harboring DJ-1 mutations display mitochondrial impairment that can be reverted by antioxidants. Increased ROS production was observed in brain and skeletal muscle mitochondria from DJ-1 knockout mice, but an exclusive ROS scavenging role for DJ-1 was unlikely. ${ }^{65}$ Recently, using transgenic mice that expressed a redox-sensitive variant of green fluorescent protein targeted to the mitochondrial matrix, Guzman and coworkers showed that the opening of the plasma membrane L-type $\mathrm{Ca}^{2+}$ channels during normal autonomous pacemaking created an oxidative stress that was specific to the nigral neurons. This oxidative stress engaged defenses that induced transient, mild mitochondrial depolarization or uncoupling.
When this response failed to efficiently occur, as in the case of DJ-1 knockout mice, neuronal damage might prevail. ${ }^{66}$ These data propose that, in mature substantia nigra pars compacta dopaminergic neurons, a basal mitochondrial oxidant stress is constitutively present, but it is not a consequence of aging, pathology, or the experimental preparation. Rather, it derives from the designated engagement of L-type $\mathrm{Ca}^{2+}$ channels during autonomous pacemaking. Substantia nigra pars compacta dopaminergic neurons lacking DJ-1 have elevated mitochondrial oxidative stress, which was not due to lowered expression of antioxidant enzymes, but diminished levels of uncoupling proteins UCP4 and UCP5. These findings added new important information on the relationship between DJ-1, mitochondrial uncoupling, and $\mathrm{Ca}^{2+}$-induced stress. ${ }^{66}$

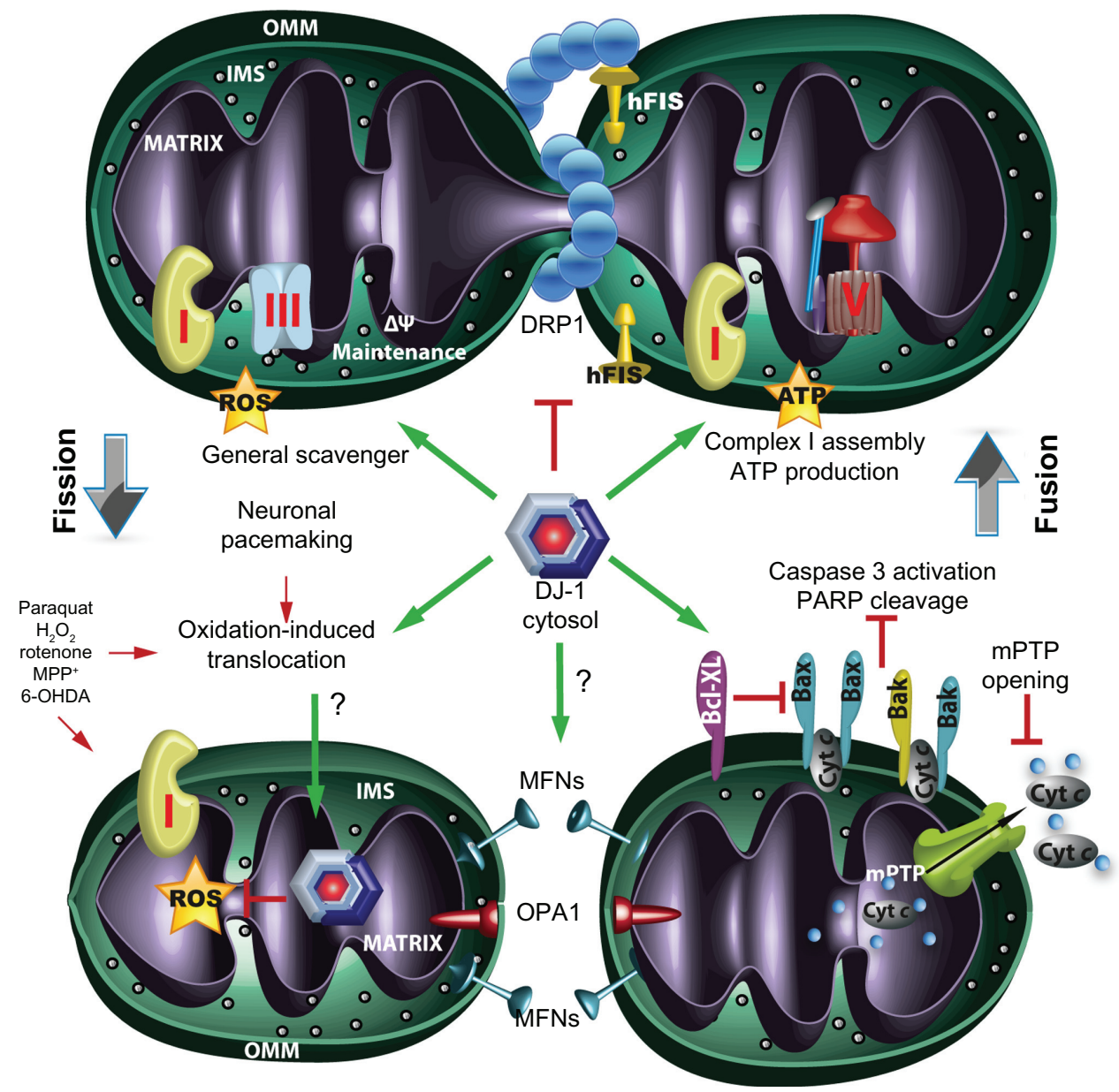

Figure $3 \mathrm{DJ}$-I multifunctional roles in the control of mitochondria-related processes.

Notes: Its protective role against reactive oxygen species (ROS) and mitochondrial toxins, as well as in preventing mitochondria-induced apoptotic death (by preventing the mPTP opening) is highlighted in this scheme. Emerging evidence also suggests that it may also regulate the process of fission/fusion by modulating the expression of the protein involved in this process, thus guaranteeing proper mitochondria morphology. Red bar-headed lines and green arrow-headed lines indicate inhibitory and stimulatory effects by DJ-I,respectively.

Abbreviations: OMM, outer mitochondrial membrane; IMS, intermembrane space; mPTP, mitochondrial permeability transition pore; $\Delta \psi$, mitochondrial membrane potential; Cytc, cytochrome c; MFNs, mitofusins; OPAI, optic-atrophy I; ROS, reactive oxygen species; hFIS, human Fission factor I; Bax, Bcl-2-associated X protein; Bak, $\mathrm{Bcl}-2$ homologous antagonist killer; Bcl-XL, B-cell lymphoma-extra large; DRPI, dynamin-related protein I; 6-OHDA, 6-hydroxydopamine. 
Figure 3 summarizes the main proposed mechanisms for DJ-1 protective action. It must be pointed out that in addition to being directly involved in the control of mitochondria bioenergetics and oxidative stress, DJ-1 also participates in the regulation of mitochondrial morphology and dynamics, a role which is shared with other PD-related proteins and will be discussed in following paragraphs. Conversely, the other PD-related proteins are also involved in protective pathways against oxidative stress. Parkin knockout mice display decreased serum antioxidant capacity and increased protein and lipid peroxidation, possibly due to the observed reduction of the amount of proteins involved in mitochondrial function or oxidative stress response. ${ }^{19}$ However, other studies reported no sign of oxidative stress or of increased sensitivity to MPTP or 6-hydroxydopamine, suggesting that parkin depletion per se was not sufficient to cause these effects and consequent Parkinsonism phenotypes. The exposure to particular conditions may be required to exacerbate parkin loss-of-function defects. PINK1 depletion was instead sufficient to make dopaminergic neurons more sensitive to MPTP treatment, and interestingly this effect was reverted by expression of parkin or DJ-1 ${ }^{67}$ The involvement of $\alpha$-syn in oxidative stress is different: its overexpression rather than its depletion has been reported to cause oxidative stress ${ }^{68}$ and cell toxicity. The latter can be prevented by DJ-1 in an oxidation-dependent manner, ${ }^{69}$ suggesting a functional interplay between these two proteins.

As for LRRK2, intriguingly it has been shown to interact with human peroxiredoxin 3 (PRDX3), a mitochondrial member of the antioxidant family of thioredoxin peroxidases. Mutations affecting the LRRK2 kinase domain significantly increase the phosphorylation of PRDX3, resulting in reduced peroxidase activity. LRRK2 mutants also stimulated mitochondrial factors involved in apoptosis and induced ROS production and oxidative burst. Interestingly, analysis of postmortem human PD patients carrying the G2019S mutation showed a marked increase in phosphorylated PRDX3 with respect to normal brain, suggesting a possible link between the enhanced kinase activity of PD-linked LRRK2 mutants and neuronal cell death. ${ }^{70}$

\section{mtDNA mutations}

The mitochondrial genome (mtDNA) encodes 13 subunits of the ETC complexes (among which seven belong to complex I) and the ribosomal and transfer RNAs necessary to support intramitochondrial protein synthesis. Due to the absence of efficient repair systems, mtDNA is particularly prone to mutations. Inherited mutations cause a variety of diseases, prevalently affecting brain and muscles, two tissues with high energy requirements. Numerous studies have demonstrated a direct correlation between the presence of somatic mtDNA mutations (which increase during aging) and sporadic forms of PD. The study of cybrid cell lines harboring mtDNA from PD patients confirmed the association between mtDNA mutations and most of the PD canonical hallmarks, such as complex I deficit, Lewy body-like inclusion, higher sensitivity to MPTP, and $\mathrm{Ca}^{2+}$ overload. ${ }^{65,71-73}$ The generation of the "MitoPark" mouse, in which the TFAM gene encoding the mitochondrial transcription factor A was selectively deleted in dopaminergic neurons, further confirmed that reduced mtDNA expression results in progressive and slow degeneration of dopaminergic neurons, which display impaired ETC activity and neuronal inclusions reminiscent of those observed in sporadic PD patients. ${ }^{45,74,75}$ In addition, dopaminergic neurons show a reduction in the mitochondrial mass compared to nondopaminergic cells, which further contributes to mitochondrial dysfunctions. Interestingly, mutations in the nuclear encoded mtDNA polymerase gamma gene impaired mtDNA replication and resulted in multiple mtDNA deletions that caused typical myopathy with progressive external ophthalmoplegia and peripheral neuropathy associated with Parkinsonism, ${ }^{76}$ thus further supporting a causative link between mtDNA defects and PD.

Although a direct association between mtDNA mutations and $\alpha$-syn, DJ-1, PINK1/parkin, and LRRK2 is missing, it can be speculated that their loss or gain of toxic function may contribute to increase mtDNA damage by increasing the risk of oxidative stress exposure. However, no direct evidence has been provided so far and, in the last 10 years, most efforts have focused on the study of their direct role on mitochondrial function rather than on the search for correlation with mtDNA mutations.

At the end of this section, it is important to mention that some interesting data support a role for parkin in mitochondria biogenesis. Parkin was shown to promote the degradation of parkin-interacting substrate (PARIS), a protein that represses the transcriptional activity of the peroxisome proliferator-activated receptor gamma-coactivator 1-alpha (PGC-1 $\alpha$ ), a master regulator of mitochondrial biogenesis. ${ }^{77}$ The inactivation of parkin, either by mutation or by environmental stress, leads to the accumulation of PARIS and the ensuing inhibition of PGC- $1 \alpha$ transcription, which in turn may reduce mitochondrial biogenesis and cause oxidative phosphorylation deficiency. Conversely, parkin promotes the specific degradation of mitochondria with mutated mtDNA, ${ }^{78}$ 
thus globally contributing to the preservation of functional mitochondrial populations.

\section{$\mathrm{Ca}^{2+}$ homeostasis}

Mitochondria, in addition to being the main powerhouse of the cells, are extremely important to the spatiotemporal tuning of the $\mathrm{Ca}^{2+}$ signal. They act as dynamic buffering system, which accumulates and releases the ion in a controlled fashion. The mitochondrial membrane potential provided by the activity of the ETC drives not only ATP production but also $\mathrm{Ca}^{2+}$ accumulation into the mitochondrial matrix through the mitochondrial $\mathrm{Ca}^{2+}$ uniporter. ${ }^{79,80}$ The reaching of the thermodynamic equilibrium is prevented by the action of two antiporters that by exchanging $\mathrm{Na}^{+}(\mathrm{NCLX})^{81}$ or $\mathrm{H}^{+}\left(\mathrm{H}^{+} / \mathrm{Ca}^{2+}\right.$ exchanger $)$ mediate the efflux of $\mathrm{Ca}^{2+}$ from the matrix.

Mitochondrial $\mathrm{Ca}^{2+}$ uptake relies on the proton electrochemical gradient, but also on the generation of microdomains of high $\mathrm{Ca}^{2+}$ concentration that match the low $\mathrm{Ca}^{2+}$ affinity of the uniporter and permit the rapid accumulation of the cation in the mitochondrial matrix. The microdomains are generated by the opening of the ER $\mathrm{Ca}^{2+}$ channels, eg, the 1,4,5 inositol-trisphosphate receptors $\left(\mathrm{InsP}_{3} \mathrm{R}\right)$ and specifically sensed by mitochondria that have established close contacts with them..$^{82}$ The tethers linking the two organelles have been physically visualized, ${ }^{83}$ and mitofusin 2 has been proven to be an essential component of these structures. ${ }^{84}$ Another important player that facilitates the transfer of $\mathrm{Ca}^{2+}$ between ER and mitochondria ${ }^{85}$ is the voltage-dependent anion channel (VDAC1), located in the OMM. Through a Grp75/mortalin-mediated coupling with $\mathrm{InsP}_{3} \mathrm{R}$, VDAC1 localizes to the so-called mitochondrial associated ERmembranes, a compartment where ER and mitochondria are juxtaposed, thus permitting physical and functional communication between them. ${ }^{86}$

A few reports have appeared proposing a general impairment of $\mathrm{Ca}^{2+}$ homeostasis as a consequence of PD-related protein loss-of-function. Increased resting cytosolic (and in some cases mitochondrial) $\mathrm{Ca}^{2+}$ levels have been documented in human neuroblastoma cells silenced for parkin expression, ${ }^{87}$ in muscle cells from DJ-1 knockout mice, ${ }^{88}$ and in cells incubated with exogenous wt or mutated $\alpha$-syn. ${ }^{89}$ In the case of $\alpha$-syn, a couple of papers proposed that its accumulation into the organelles increased mitochondrial $\mathrm{Ca}^{2+}$ levels and nitric oxide production, which have deleterious consequences such as oxidative stress and cytochrome $c$ release ${ }^{68,90}$ All these observations suggest that the loss of function of these proteins may account for $\mathrm{Ca}^{2+}$ overload that in turn could make the cells more susceptible to other stress insults. However, no evidence was provided on the possible mechanism of this action, except for $\alpha$-syn, which was proposed to increase plasma membrane $\mathrm{Ca}^{2+}$ permeability ${ }^{89}$ or modulate L-type $\mathrm{Ca}^{2+}$ channel activity. ${ }^{91,92}$ Studies on the possible involvement of PD-related proteins in the mitochondrial $\mathrm{Ca}^{2+}$ metabolism were absent, with the exception of PINK1, even though contradictory results have been reported by different groups (see below). The possibility that in physiological conditions the PD-related proteins may modulate $\mathrm{Ca}^{2+}$ signaling has never been considered persistently. We have recently found that $\alpha$-syn, ${ }^{93}$ parkin, ${ }^{94}$ and DJ- $1{ }^{95}$ share a common role in modulating mitochondrial $\mathrm{Ca}^{2+}$ signaling: their overexpression in model cells augments mitochondrial $\mathrm{Ca}^{2+}$ transients generated by the $\mathrm{Ca}^{2+}$ release from the intracellular stores following cell stimulation. Interestingly, this action was due to a common role in enhancing ER-mitochondria tethering, thus favoring ER-mitochondria $\mathrm{Ca}^{2+}$ transfer following the opening of the ER $\mathrm{Ca}^{2+}$-release $\mathrm{InsP}_{3}$ channels. A deeper analysis of the consequences of this effect revealed that this action is required to maintain proper mitochondrial morphology, since it guarantees essential regulation of cell bioenergetics, as previously documented. ${ }^{96}$ In the case of parkin, we have also found that the enhancement of ER-mitochondria coupling represents a bioenergetic advantage: ATP production in stimulated parkin-overexpressing cells was increased. ${ }^{94} \mathrm{The}$ case of $\alpha$-syn overexpression was also interesting: relatively low levels of $\alpha$-syn protein, which maintained it in a soluble state, favored ER-mitochondria communication, but high expression levels induced $\alpha$-syn redistribution to cytosolic foci and subtracted the cytosolic pool from its role in enhancing ER-mitochondria contact sites. As a consequence, the ability of mitochondria to take up $\mathrm{Ca}^{2+}$ was impaired and autophagy response was activated ${ }^{93}$ Altogether, these data suggest that defective ER-mitochondria communication not only may represent a common hint in determining mitochondrial impairment but may also be the starting point for the development of cell degeneration.

As for the possible involvement of PINK1 and LRRK2 in mitochondrial $\mathrm{Ca}^{2+}$ homeostasis, some data have appeared in the last 5 years. A couple of papers pointed out the possibility that PINK1 may regulate $\mathrm{Ca}^{2+}$ efflux from the mitochondria, suggesting that PINK1-deficient cells or cells expressing mutant PINK1 may undergo $\mathrm{Ca}^{2+}$ overload and ROS production, with consequent loss of energy-supplying substrates and impaired respiration. ${ }^{97,98}$ Another recent paper proposed that PINK1 deficiency induces moderate mitochondrial depolarization, which results in reduced mitochondrial $\mathrm{Ca}^{2+}$ uptake, and as a consequence impaired mitochondrial ATP synthesis. ${ }^{99}$ Thus, the scenario is still unclear and demands further investigation. 
Very recently, it has been reported that the expression of mutant forms of LRRK2 in mouse cortical neurons enhanced autophagic degradation of mitochondria and dendrite shortening. Interestingly, $\mathrm{Ca}^{2+}$ chelators or inhibitors of voltage-gated $\mathrm{L}$-type $\mathrm{Ca}^{2+}$ channels prevented mitochondrial degradation, suggesting that these effects are due a deficit in $\mathrm{Ca}^{2+}$-buffering capacity. ${ }^{100}$ Mitochondria $\mathrm{Ca}^{2+}$-buffering action has been proposed as a mechanism to counteract axonal degeneration of dopaminergic neurons, one of the first signs of neurodegeneration that precedes cell-body loss, and the $\mathrm{NAD}^{+}$adenylyltransferase (NMAT) biosynthetic enzyme has been shown to prevent axonal-induced degeneration by enhancing mitochondria $\mathrm{Ca}^{2+}$ buffering and motility. ${ }^{101}$ However, the mechanisms of action are still obscure, and the direct involvement of mitochondria is not unequivocally recognized. ${ }^{102} \mathrm{~A}$ very recent paper has proposed that NMAT-mediated cytoprotection in yeast is dependent on the targeting of misfolded or aggregated proteins, ie, $\alpha$-syn, to the proteasome pathway, ${ }^{103}$ rather than on the participation of mitochondria. Further studies will be necessary to define the precise pathways involved and to establish a solid link between NMAT-mediated protection and PD. In this direction, encouraging data have shown that the "Wallerian degeneration slow" fusion protein, composed of 70 amino acids of the ubiquitination factor $\mathrm{E} 4 \mathrm{~b}$, a linker region, and the coding sequence for NMAT1, protects dopaminergic neurites from $\mathrm{MPP}^{+}$neurotoxicity. ${ }^{104}$

\section{Mitochondria dynamics}

According to the canonical textbooks, mitochondria are bean-shaped double-membrane organelles found in all the eukaryotes and vary in number and location in different cell types. This view might suggest that they are isolated and static organelles that nevertheless are capable of providing required energy to all the cell subcompartments. Mitochondria do not possess cables for the delivery of such energy to the different subcellular districts that in some cases could also be far from the cell body where they are mainly located. To compensate for this lack, they have developed the ability to directly reach the sites of energy requirement by themselves, moving along predefined cellular highways. The updated description of mitochondria corresponds to a highly interconnected network distributed through the entire volume of the cell, able to dynamically undergo cycles of fission and fusion that, together with the transport along microtubule tracks and motor proteins, guarantee mitochondrial positioning to the sites where their action is required. In the last few years, increasing evidence has been provided on the causative link between the dysregulation of mitochondria movements and $\mathrm{PD}$, thus reinforcing the role of mitochondrial dysfunction in PD pathogenesis. In the following paragraphs, we will dissect the pathophysiological aspects of these processes.

\section{Fission/fusion}

Mitochondria dynamics guarantee many biological functions. Mitochondrial fission is important during cell division for partitioning and distributing the organelles, for the release of proapoptotic factors, and for the turnover of damaged organelles. Mitochondrial fusion is fundamental to improving cellular bioenergetics and to counteracting impairment in respiratory functions by complementing defective mtDNA gene products. As cited above, under physiological conditions, the mitochondria network is continuously engaged in processes of fission and fusion. In mammals, these processes are governed by GTPase proteins and a few adaptor proteins anchored on the mitochondrial membranes collectively referred to as mitochondria-shaping proteins. ${ }^{105}$ The main players in mitochondrial fission are the dynamin-related protein DRP1, Fis1, and the OMM adaptor protein mitochondrial fission factor (MFF). DRP1 is a cytosolic GTPase protein ${ }^{106}$ that during mitochondrial fission and upon dephosphorylation by calcineurin ${ }^{107}$ is recruited on mitochondria, where it interacts with its putative adaptors and upon oligomerization forms ring-shaped structures that induce mitochondrial constriction and consequent fission. ${ }^{108}$ Fis 1 is a tetratricopeptide domain-containing protein, and it was initially considered the DRP1 receptor at the OMM; ${ }^{109}$ however, the finding that its overexpression promotes mitochondrial fragmentation without affecting DRP1 translocation to mitochondria indicates that additional pathways for DRP1 recruitment may exist. ${ }^{110}$ The mitochondrial tail-anchored OMM protein MFF is currently the best-acknowledged receptor for DRP1: its depletion attenuates mitochondrial division, both in mammalian and Drosophila cells, and importantly reduces the amount of DRP1 that is recruited to mitochondria. ${ }^{111}$ Interestingly, it has been shown that mitochondrial fission events predominantly occur at specific sites where some ER tubules wrap around mitochondria, and that DRP1 and MFF localize at these contact sites, ${ }^{112}$ thus enforcing the notion that MFF-mediated DRP1 recruitment on the OMM is an essential step. The fusion mechanism is governed by the dynamin-related GTPases named mitofusins (MFN1 and MFN2) and the optic atrophy protein OPA1, which mediate the fusion of the OMM and the IMM, respectively. ${ }^{113}$ 
In addition to participating to the process of mitochondria fusion, as already mentioned, MFN2 is an essential constituent in ER-mitochondria tethering, with important implications in $\mathrm{Ca}^{2+}$ signaling and lipid metabolism as well. ${ }^{84}$ The identification of the aforementioned proteins and the elucidation of the molecular processes involved in governing mitochondria dynamics make evident that the dysregulation of these pathways is a common feature in several neurodegenerative conditions, including PD. ${ }^{114}$

However, it is still unclear whether the dysfunctions are the cause or the consequence of the pathological condition. In this respect, the study of the function of the gene products involved in familial forms of PD has contributed to clarifying some aspects. Mitochondrial fragmentation and impairment of mitochondria trafficking and of their clearance have been observed in different cell types harboring reduced levels of expression, or mutations, in any of the PD-related proteins. More compelling evidence has been provided for DJ-1, PINK1, and parkin, but recently, a tight relationship between LRRK2 and $\alpha$-syn and the maintenance of mitochondria morphology has also emerged. Thus, even if the pathways discussed in the first part of this review have been traditionally considered important in PD etiopathogenesis, new emerging data suggest a strong link between the loss of function of PD-related proteins and the dysregulation of the expression and/or activity of DRP1 and Fis1 proteins, ${ }^{115-118}$ thus underlining the relevance of the impairment of mitochondria dynamics.

PINK1 and parkin have been shown to be master regulators of a process named mitophagy, which is in place to preserve mitochondria network functionality by selective removal of dysfunctional mitochondria (see below). This process is strictly connected with the fission/fusion pathway. Studies in parkin- or PINK1-deficient mammalian cells have reported variable effects on mitochondrial morphology, suggesting both a pro-fission and a pro-fusion action on mitochondria for both of the proteins. ${ }^{119}$ Further evidence supports the notion that depending on different cell status, PINK1/parkin could play a dual role, acting as pro-fusion factors to maintain basal mitochondrial wellness and as pro-fission factors to get rid of dysfunctional cellular products and to improve energetic status under conditions of mitochondrial impairment. ${ }^{120,121}$

As for DJ-1, its silencing has been shown to induce mitochondria fragmentation; however, if general agreement has been reached on the final outcome, the mechanism is still controversial. Some authors have observed an increase in DRP1 expression as well as enhanced DRP1 translocation to mitochondria, others have instead excluded that the mechanism is DRP1-mediated and rather proposed that a reduction of MFN1 expression may be determinant ${ }^{115,122-124}$ (Figure 3). We have recently shown that the overexpression of DJ-1 in HeLa cells completely counteracted p53-induced alteration of mitochondrial morphology by restoring the proper ER-mitochondria tethering and $\mathrm{Ca}^{2+}$ transfer, independently on the DRP1- or MFN-mediated pathway. ${ }^{95}$

Many studies have reported evidence on the pro-fission role of $\alpha$-syn after its overexpression (wt as well as A30P and A53T mutants) and suggested that the effects of $\alpha$-syn on mitochondrial fragmentation may be dose-dependent. It has been shown to act independently from the modulation of the fusion/fission proteins, ${ }^{93,125}$ and also by increasing DRP1 mitochondrial translocation in an extracellular signal-regulated kinase (ERK)-dependent manner. ${ }^{36}$ We have found that $\alpha$-syn downregulation in HeLa cells impaired the mitochondrial network, ${ }^{93}$ and as mentioned earlier, we have proposed that endogenous $\alpha$-syn levels are required to maintain ER-mitochondria $\mathrm{Ca}^{2+}$ transfer that guarantees proper mitochondria morphology and physiology. Interestingly, recent studies have proposed an association between increased PD risk and the presence of a specific RNA transcript isoform of $\alpha$-syn with a longer $3^{\prime}$ untranslated region. This $3^{\prime}$ tail was supposed to increase $\alpha$-syn stability/accumulation, and interestingly its mitochondrial translocation, ${ }^{126}$ thus supporting the hypothesis that mitochondrial $\alpha$-syn localization may be at the basis of the observed phenotypes. As for LRRK2, its role in promoting mitochondrial fission has been recently proposed and confirmed by two independent groups. They have shown that the direct association of LRRK2 with DRP1 increased mitochondrial levels of DRP1 and that LRRK2 pathogenic mutations further exacerbated this interaction, which appeared to be dependent on LRRK2 activity. ${ }^{117,127}$

\section{Motility}

As cited above, mitochondria move along cytoskeletal tracks to carry "energy packs" to the sites of high demand and/ or to locate where their $\mathrm{Ca}^{2+}$-buffering action is required. Mitochondria mobility is essential in neurons to cover the long distances from the main cell body to the synapses and thus supply energy to these highly demanding districts. Thanks to the action of different molecular motors, organelle movement occurs in both directions: towards the cell periphery (kinesin-dependent anterograde transport) and to the nucleus (dynein-mediated retrograde transport). By bridging to kinesins, the adaptor proteins Milton and Miro mediate the anchorage of mitochondria to the microtubule 
network, allowing their anterograde transport. Miro proteins (Miro1 and Miro2 in humans) are well-conserved mitochondrial Ras GTPases with a C-terminus domain containing a transmembrane portion, which is responsible for their anchoring to the OMM. The $\mathrm{C}$ terminus also contains two $\mathrm{Ca}^{2+}$-binding EF-hand motifs that confer $\mathrm{Ca}^{2+}$ sensitivity to the mitochondria trafficking process. Milton proteins are cytosolic and link Miro to kinesin. The retrograde axonal transport is under the control of dynein motors and is functionally coupled with kinesins that are required to transport dyneins to the periphery of cells. Mitochondria, similarly to other axonally transported organelles, can also move along actin filaments. This transport involves myosin motors, but the role of this short-range movement is less clear. The relationship between impaired mitochondria trafficking and PD pathogenesis is still largely unexplored, but the identification of a mitochondrial multiprotein complex that contains PINK1, Miro, and Milton led to the seminal observation that PINK1 activity may regulate mitochondrial trafficking. ${ }^{128}$ Recently, two independent groups have confirmed this hypothesis, showing that PINK1-mediated phosphorylation of Miro represents a signal for the recruitment of parkin at mitochondria and consequent Miro degradation, with successive detachment of mitochondria from microtubules. ${ }^{129,130}$ Consistently with these findings, overexpression of PINK1 inhibited axonal mitochondria transport in both anterograde and retrograde directions in Drosophila dopaminergic neurons. ${ }^{130}$ Analogously, DJ-1 knockdown significantly reduced mitochondrial motility in cellular processes of astrocyte-enriched cultures, ${ }^{131}$ and $\alpha$-syn overexpression impaired the movement of mitochondria in both SH-SY5Y neuroblastoma cells and hippocampal neurons from transgenic mice, this effect being particularly prominent in the case of A53T $\alpha$-syn mutant overexpression. ${ }^{132}$ Interestingly instead, the expression of LRRK2 mutants did not elicit defects in mitochondrial trafficking, but caused mitochondrial depolarization and reduced $\mathrm{Ca}^{2+}$-buffering capacity. Mouse cortical neurons expressing mutant LRRK2 exhibited autophagic degradation of mitochondria and dendrite shortening, in agreement with the finding that mitochondrial content/function is essential for dendritic morphogenesis and maintenance. ${ }^{100}$

\section{Mitophagy}

Mitophagy, the process developed to selectively remove dysfunctional mitochondria, has emerged as essential to guaranteeing the presence of functional mitochondria networks in the cells. ${ }^{133}$ This quality-control process shares some molecular components with the (macro)autophagy pathway. Its link with PD originated from the observation that mitochondrial membrane potential disruption by mitochondria-damaging agents, such as carbonyl cyanide $m$-chlorophenylhydrazone, induced parkin translocation to depolarized mitochondria and promoted their LC3-mediated autophagic elimination in mammalian cells. ${ }^{23}$ Since then, numerous independent research groups have exhaustively investigated the mechanistic insights of this process, identifying the pathways involved both in the tagging and in the recognition of dysfunctional mitochondria, and the proteins that participate to their selective degradation. However, there are still many open questions. Among them, the most prominent is whether these mechanisms are effectively acting in neurons, and which are the physiopathological signals that drive parkin recruitment to mitochondria and trigger the selective removal of the damaged population. The current view is summarized in Figure 4. PINK1 endogenous levels are kept very low by complex posttranslational processing that involves PINK1 mitochondrial import (mediated by an N-terminal mitochondrial targeting signal) and several proteases. Among them, the presenilin associated rhomboid-like protein generates a PINK1 soluble form, which is released to the cytoplasm and in healthy mitochondria removed by a proteasome-dependent pathway. However, in the case of membrane potential-deficient mitochondria, mitochondrial import and PINK1 processing are impaired. PINK1 accumulates on the OMM, where it recruits parkin ${ }^{134}$ that in turn mediates the ubiquitination of a number of OMM proteins, among them MFNs, ${ }^{135,136}$ DRP $1,{ }^{116}$ VDAC $1,{ }^{137}$ Miro, ${ }^{129}$ and Bcl-2. ${ }^{138}$ The resulting accumulation of polyubiquitinated proteins may recruit the ubiquitin-binding and LC3-binding adaptor protein p $62^{137}$ and the ubiquitin-binding deacetylase HDAC6, ${ }^{139}$ leading to perinuclear clustering of mitochondria. Finally, this is followed by engulfment of the damaged mitochondria by autophagosomes and subsequent lysosomal degradation. How PINK1 recruits cytosolic parkin to the mitochondria and parkin participates in the formation of "mitophagosome" is still unclear. PINK1 kinase activity is required for parkin recruitment to the mitochondrial surface, where parkin's E3 activity appears to be stimulated. ${ }^{24,140}$ It has been demonstrated that PINK1 autophosphorylation at Ser228 and Ser402 residues (a process hindered in most of the PD-relevant mutations) is essential to promote parkin recruitment. ${ }^{141}$

Very recently, it has been shown that PINK1 phosphorylates MFN2 that, in turn, acts as a parkin receptor on the OMM. This study proposes that MFN2, in addition to acting downstream of parkin in the activation of the mitophagy 


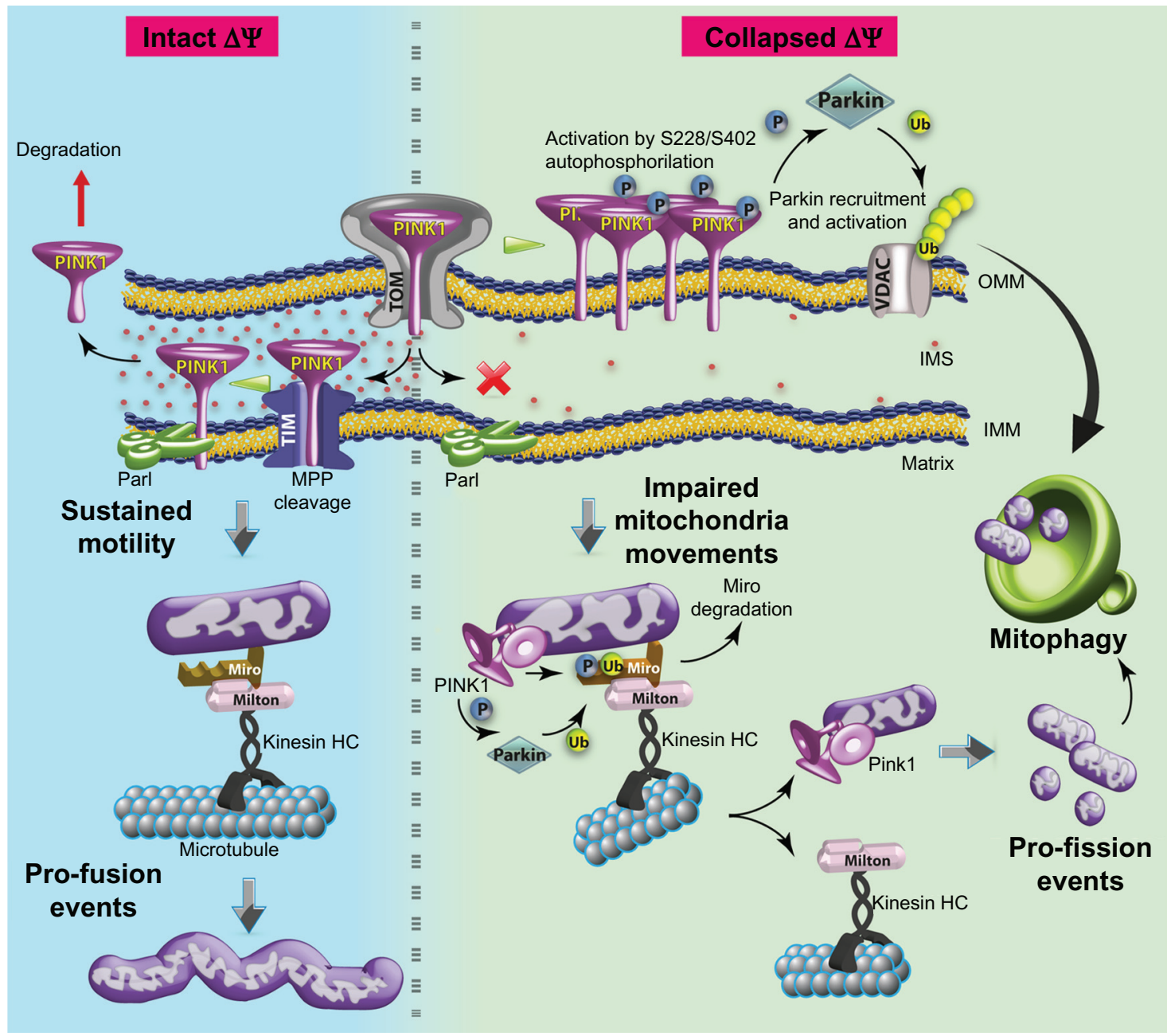

Figure 4 PINKI and parkin action on mitochondria.

Notes: PINKI/parkin are involved in the selective removal of damaged mitochondria by mitophagy. They also participate in the regulation of fission/fusion processes and mitochondria movements. PINKI accumulation at the mitochondrial outer membrane acts as a tag to recruit parkin to dysfunctional mitochondria and activate its ubiquitinligase activity (see the text for further details).

Abbreviations: $\Delta \psi$, mitochondrial membrane potential, OMM, outer mitochondrial membrane; IMS, intermembrane space; IMM, inner mitochondrial membrane; VDAC, voltage-dependent anion channel; PINKI, PTEN-induced kinase I; Parl, presenilin-associated rhomboid-like protein; MPP, mitochondrial processing peptidase; Kinesin HC, kinesin heavy chain; Ub, ubiquitin.

process, may also act upstream to translate PINK1 signal for parkin translocation. ${ }^{142}$ Besides the direct involvement of PINK1/parkin pathway in the regulation of mitophagy, a possible involvement of $\alpha$-syn is emerging, even if its precise role is still elusive. In general, $\alpha$-syn overexpression results in impairment of proteasomal degradation and lysosomal autophagy, including chaperone-mediated autophagy, ${ }^{143}$ thus compromising the removal of misfolded or damaged polypeptides. Interestingly, however, mitophagy activity was increased in an $\alpha$-syn-induced toxicity condition. ${ }^{55,144}$ Similarly, the direct involvement of DJ-1 in the process of mitophagy is still largely unexplored. Some reports have shown that DJ-1 silencing resulted both in upregulation ${ }^{123,124}$ and downregulation ${ }^{122}$ of the autophagy process. The neuroprotective effect of DJ-1 on rotenone-induced injury of rat dopaminergic neurons and cultured cells was mediated by the enhancement in ERK-dependent mitophagy. ${ }^{145}$ The loss of DJ-1 function resulted in increased stress-induced parkin recruitment and mitophagy. These findings strongly suggest a link between DJ-1, the PINK1/parkin pathway, oxidative stress, and mitophagy in neurons. ${ }^{146}$ As for LRRK2, its link with mitochondria function is less evident in respect of the other PD-related proteins; however, recent investigations on brain cortex of transgenic mice expressing mutant LRRK2 have shown increased density of autophagic vacuoles. ${ }^{61}$ A study performed on cell cultures either overexpressing mutant LRRK2 or silenced for its expression revealed that LRRK2 acts both as positive and negative regulator of autophagy, thus suggesting that its relationship with this degradation pathway is still unclear. ${ }^{147}$ 


\section{Concluding remarks}

For years, defects in complex I activity and oxidative stress have been considered the main actors in the pathogenesis of PD. The identification and the characterization of familial PD forms associated with mutations in specific proteins, together with the development of cell and genetic animal models, are making their acknowledged primary role in causing defects in bioenergetics far less compelling. The study of the biological function of the PD-related proteins has revealed that mitochondria quality control is an essential step to guarantee proper mitochondria physiology. Perturbed mitochondria dynamics and mitochondria-ER cross talk are now emerging as common causative determinants for familial and sporadic PD. At advanced stages, both of them may result in bioenergetic defects that finally compromise cell survival.

Numerous questions have still to be solved to unravel mechanistic insights. Among them, one of the most prominent from the mitochondrial point of view is to reconcile the results obtained in several different cellular models with the in vivo situation. Another important aspect to be deeply explored is whether mitochondrial dysfunction could represent a site of intervention early in the pathogenic process, as suggested in recent years..$^{70,148}$ The big progress that has been made on the role of mitochondria function in the pathogenesis of PD strongly encourages pursuing this possibility.

\section{Acknowledgments}

Work performed by the authors on the topic was supported over the years by grants to MB from the Italian Ministry of University and Research PRIN 2008 and the local founding of the University of Padova (Progetto di Ateneo 2008 CPDA082825). The authors apologize to colleagues whose work has not been cited due to space limitations.

\section{Disclosure}

The authors report no conflicts of interest in this work.

\section{References}

1. Spillantini MG, Schmidt ML, Lee VM, Trojanowski JQ, Jakes R, Goedert M. Alpha-synuclein in Lewy bodies. Nature. 1997;388: 839-840.

2. Langston JW, Ballard P, Tetrud JW, Irwin I. Chronic Parkinsonism in humans due to a product of meperidine-analog synthesis. Science. 1983;219:979-980.

3. Sherer TB, Betarbet R, Testa CM, et al. Mechanism of toxicity in rotenone models of Parkinson's disease. J Neurosci 2003;23:10756-10764.

4. Exner N, Lutz AK, Haass C, Winklhofer KF. Mitochondrial dysfunction in Parkinson's disease: molecular mechanisms and pathophysiological consequences. EMBO J. 2012;31:3038-3062.
5. Schapira AH. Mitochondrial pathology in Parkinson's disease. Mt Sinai J Med. 2011;78:872-881.

6. Hardy J, Lewis P, Revesz T, Lees A, Paisan-Ruiz C. The genetics of Parkinson's syndromes: a critical review. Curr Opin Gen Dev. 2009;19: 254-265.

7. Lesage S, Brice A. Parkinson's disease: from monogenic forms to genetic susceptibility factors. Hum Mol Genet. 2009;18:R48-R59.

8. Valente EM, Abou-Sleiman PM, Caputo V, et al. Hereditary earlyonset Parkinson's disease caused by mutations in PINK1. Science. 2004;304:1158-1160.

9. Wood-Kaczmar A, Gandhi S, Yao Z, et al. PINK1 is necessary for long term survival and mitochondrial function in human dopaminergic neurons. PLoS One. 2008;3:e2455.

10. Pridgeon JW, Olzmann JA, Chin LS, Li L. PINK1 protects against oxidative stress by phosphorylating mitochondrial chaperone TRAP1. PLoS Biol. 2007;5:e172.

11. Gautier CA, Kitada T, Shen J. Loss of PINK1 causes mitochondrial functional defects and increased sensitivity to oxidative stress. Proc Natl Acad Sci U S A. 2008;105:11364-11369.

12. Morais VA, Verstreken P, Roethig A, et al. Parkinson's disease mutations in PINK1 result in decreased complex I activity and deficient synaptic function. EMBO Mol Med. 2009;1:99-111.

13. Ashrafi G, Schwarz TL. The pathways of mitophagy for quality control and clearance of mitochondria. Cell Death Differ. 2013;20:31-42.

14. Youle RJ, Narendra DP. Mechanisms of mitophagy. Nat Rev Mol Cell Biol. 2011;12:9-14.

15. Deng H, Dodson MW, Huang H, Guo M. The Parkinson's disease genes pink1 and parkin promote mitochondrial fission and/or inhibit fusion in Drosophila. Proc Natl Acad Sci U S A. 2008;105:14503-14508.

16. Park J, Lee G, Chung J. The PINK1-Parkin pathway is involved in the regulation of mitochondrial remodeling process. Biochem Biophys Res Commun. 2009;378:518-523.

17. Yang Y, Ouyang Y, Yang L, et al. Pink1 regulates mitochondrial dynamics through interaction with the fission/fusion machinery. Proc Natl Acad Sci U S A. 2008;105:7070-7075.

18. Poole AC, Thomas RE, Andrews LA, McBride HM, Whitworth AJ, Pallanck LJ. The PINK1/Parkin pathway regulates mitochondrial morphology. Proc Natl Acad Sci U S A. 2008;105:1638-1643.

19. Palacino JJ, Sagi D, Goldberg MS, et al. Mitochondrial dysfunction and oxidative damage in parkin-deficient mice. J Biol Chem. 2004;279:18614-18622.

20. Jiang H, Ren Y, Zhao J, Feng J. Parkin protects human dopaminergic neuroblastoma cells against dopamine-induced apoptosis. Hum Mol Genet. 2004;13:1745-1754.

21. Darios F, Corti O, Lucking CB, et al. Parkin prevents mitochondrial swelling and cytochrome $\mathrm{c}$ release in mitochondria-dependent cell death. Hum Mol Genet. 2003;12:517-526.

22. Petrucelli L, O'Farrell C, Lockhart PJ, et al. Parkin protects against the toxicity associated with mutant alpha-synuclein: proteasome dysfunction selectively affects catecholaminergic neurons. Neuron 2002;36:1007-1019.

23. Narendra D, Tanaka A, Suen DF, Youle RJ. Parkin is recruited selectively to impaired mitochondria and promotes their autophagy. $J$ Cell Biol. 2008; 183:795-803.

24. Matsuda N, Sato S, Shiba K, et al. PINK1 stabilized by mitochondrial depolarization recruits Parkin to damaged mitochondria and activates latent Parkin for mitophagy. J Cell Biol. 2010;189:211-221.

25. Blackinton J, Ahmad R, Miller DW, et al. Effects of DJ-1 mutations and polymorphisms on protein stability and subcellular localization. Brain Res Mol Brain Res. 2005;134:76-83.

26. Canet-Aviles RM, Wilson MA, Miller DW, et al. The Parkinson's disease protein DJ-1 is neuroprotective due to cysteine-sulfinic acid-driven mitochondrial localization. Proc Natl Acad Sci U S A. 2004;101: 9103-9108.

27. Li HM, Niki T, Taira T, Iguchi-Ariga SM, Ariga H. Association of DJ-1 with chaperones and enhanced association and colocalization with mitochondrial Hsp70 by oxidative stress. Free Radic Res. 2005;39:1091-1099. 
28. Maita C, Maita H, Iguchi-Ariga SM, Ariga H. Monomer DJ-1 and its $\mathrm{N}$-terminal sequence are necessary for mitochondrial localization of DJ-1 mutants. PLoS One. 2013;8:e54087.

29. Zhang L, Shimoji M, Thomas B, et al. Mitochondrial localization of the Parkinson's disease related protein DJ-1: implications for pathogenesis. Hum Mol Genet. 2005;14:2063-2073.

30. Andres-Mateos E, Perier C, Zhang L, et al. DJ-1 gene deletion reveals that DJ-1 is an atypical peroxiredoxin-like peroxidase. Proc Natl Acad Sci U S A. 2007;104:14807-14812.

31. Taira T, Saito Y, Niki T, Iguchi-Ariga SM, Takahashi K, Ariga H. DJ-1 has a role in antioxidative stress to prevent cell death. EMBO Rep. 2004;5:213-218.

32. Blackinton J, Lakshminarasimhan M, Thomas KJ, et al. Formation of a stabilized cysteine sulfinic acid is critical for the mitochondrial function of the parkinsonism protein DJ-1. J Biol Chem. 2009;284: 6476-6485.

33. Bonifati V, Rizzu P, Squitieri F, et al. DJ-1(PARK7), a novel gene for autosomal recessive, early onset parkinsonism. Neurol Sci. 2003;24: 159-160.

34. Burré J, Sharma M, Tsetsenis T, Buchman V, Etherton MR, SüdhofTC. Alpha-synuclein promotes SNARE-complex assembly in vivo and in vitro. Science. 2010;329:1663-1667.

35. Nemani VM, Lu W, Berge V, et al. Increased expression of alphasynuclein reduces neurotransmitter release by inhibiting synaptic vesicle reclustering after endocytosis. Neuron. 2010;65:66-79.

36. Kamp F, Exner N, Lutz AK, et al. Inhibition of mitochondrial fusion by alpha-synuclein is rescued by PINK1, Parkin and DJ-1. EMBO J. 2010;29:3571-3589.

37. Cole NB, Dieuliis D, Leo P, Mitchell DC, Nussbaum RL. Mitochondrial translocation of alpha-synuclein is promoted by intracellular acidification. Exp Cell Res. 2008;314:2076-2089.

38. Lin CH, Tsai PI, Wu RM, Chien CT. LRRK2 Parkinson's disease: from animal models to cellular mechanisms. Rev Neurosci. 2011;22: 411-418.

39. Cookson MR. The role of leucine-rich repeat kinase 2 (LRRK2) in Parkinson's disease. Nat Rev Neurosci. 2010;11:791-797.

40. Greggio E, Cookson MR. Leucine-rich repeat kinase 2 mutations and Parkinson's disease: three questions. ASN Neuro. 2009;1:e0002.

41. West AB, Moore DJ, Biskup S, et al. Parkinson's disease-associated mutations in leucine-rich repeat kinase 2 augment kinase activity. Proc Natl Acad Sci U S A. 2005;102:16842-16847.

42. Biskup S, Moore DJ, Celsi F, et al. Localization of LRRK2 to membranous and vesicular structures in mammalian brain. Ann Neurol. 2006;60:557-569

43. Mitchell P. Coupling of phosphorylation to electron and hydrogen transfer by a chemi-osmotic type of mechanism. Nature. 1961;191:144-148.

44. Gegg ME, Cooper JM, Schapira AH, Taanman JW. Silencing of PINK1 expression affects mitochondrial DNA and oxidative phosphorylation in dopaminergic cells. PLoS One. 2009;4:e4756.

45. Kim KH, Song K, Yoon SH, Shehzad O, Kim YS, Son JH. Rescue of PINK1 protein null-specific mitochondrial complex IV deficits by ginsenoside Re activation of nitric oxide signaling. J Biol Chem. 2012;287: 44109-44120.

46. Vilain S, Esposito G, Haddad D, et al. The yeast complex I equivalent NADH dehydrogenase rescues pink1 mutants. PLoS Genet. 2012;8:e1002456.

47. Mortiboys H, Thomas KJ, Koopman WJ, et al. Mitochondrial function and morphology are impaired in parkin-mutant fibroblasts. Ann Neurol. 2008; $64: 555-565$.

48. Grunewald A, Voges L, Rakovic A, et al. Mutant Parkin impairs mitochondrial function and morphology in human fibroblasts. PLoS One. 2010;5:e12962.

49. Hayashi T, Ishimori C, Takahashi-Niki K, et al. DJ-1 binds to mitochondrial complex I and maintains its activity. Biochem Biophys Res Commun. 2009;390:667-672.

50. Kwon HJ, Heo JY, Shim JH, et al. DJ-1 mediates paraquat-induced dopaminergic neuronal cell death. Toxicol Lett. 2011;202:85-92.
51. Mullett SJ, Hinkle DA. DJ-1 deficiency in astrocytes selectively enhances mitochondrial complex I inhibitor-induced neurotoxicity. J Neurochem. 2011;117:375-387.

52. Heo JY, Park JH, Kim SJ, et al. DJ-1 null dopaminergic neuronal cells exhibit defects in mitochondrial function and structure: involvement of mitochondrial complex I assembly. PLoS One. 2012;7:e32629.

53. Ellis CE, Murphy EJ, Mitchell DC, et al. Mitochondrial lipid abnormality and electron transport chain impairment in mice lacking alphasynuclein. Mol Cell Biol. 2005;25:10190-10201.

54. Devi L, Raghavendran V, Prabhu BM, Avadhani NG, Anandatheerthavarada HK. Mitochondrial import and accumulation of alpha-synuclein impair complex I in human dopaminergic neuronal cultures and Parkinson disease brain. J Biol Chem. 2008;283:9089-9100.

55. Chinta SJ, Mallajosyula JK, Rane A, Andersen JK. Mitochondrial alphasynuclein accumulation impairs complex I function in dopaminergic neurons and results in increased mitophagy in vivo. Neurosci Lett. 2010;486:235-239.

56. Loeb V, Yakunin E, Saada A, Sharon R. The transgenic overexpression of alpha-synuclein and not its related pathology associates with complex I inhibition. J Biol Chem. 2010;285:7334-7343.

57. Liu G, Zhang C, Yin J, et al. Alpha-synuclein is differentially expressed in mitochondria from different rat brain regions and dose-dependently down-regulates complex I activity. Neurosci Lett. 2009;454:187-192.

58. Elkon H, Don J, Melamed E, Ziv I, Shirvan A, Offen D. Mutant and wild-type alpha-synuclein interact with mitochondrial cytochrome $\mathrm{C}$ oxidase. J Mol Neurosci. 2002;18:229-238.

59. Banerjee K, Sinha M, Pham Cle L, et al. Alpha-synuclein induced membrane depolarization and loss of phosphorylation capacity of isolated rat brain mitochondria: implications in Parkinson's disease. FEBS Lett. 2010;584:1571-1576.

60. Martin LJ, Pan Y, Price AC, et al. Parkinson's disease alpha-synuclein transgenic mice develop neuronal mitochondrial degeneration and cell death. J Neurosci. 2006;26:41-50.

61. Ramonet D, Daher JP, Lin BM, et al. Dopaminergic neuronal loss, reduced neurite complexity and autophagic abnormalities in transgenic mice expressing G2019S mutant LRRK2. PLoS One. 2011;6:e18568.

62. Mortiboys H, Johansen KK, Aasly JO, Bandmann O. Mitochondrial impairment in patients with Parkinson disease with the G2019S mutation in LRRK2. Neurology. 2010;75:2017-2020.

63. Andres-Mateos E, Mejias R, Sasaki M, et al. Unexpected lack of hypersensitivity in LRRK2 knock-out mice to MPTP (1-methyl4-phenyl-1, 2,3,6-tetrahydropyridine). J Neurosci. 2009;29: 15846-15850.

64. Surmeier DJ, Schumacker PT. Calcium, bioenergetics and neuronal vulnerability in Parkinsons disease. J Biol Chem. Epub October 19, 2012.

65. Giaime E, Yamaguchi H, Gautier CA, Kitada T, Shen J. Loss of DJ-1 does not affect mitochondrial respiration but increases ROS production and mitochondrial permeability transition pore opening. PLoS One. 2012;7:e40501.

66. Guzman JN, Sanchez-Padilla J, Wokosin D, et al. Oxidant stress evoked by pacemaking in dopaminergic neurons is attenuated by DJ-1. Nature. 2010;468:696-700.

67. Haque ME, Mount MP, Safarpour F, et al. Inactivation of Pink1 gene in vivo sensitizes dopamine-producing neurons to 1-methyl-4-phenyl1,2,3,6-tetrahydropyridine (MPTP) and can be rescued by autosomal recessive Parkinson disease genes, Parkin or DJ-1. J Biol Chem. 2012;287:23162-23170.

68. Parihar MS, Parihar A, Fujita M, Hashimoto M, Ghafourifar P. Mitochondrial association of alpha-synuclein causes oxidative stress. Cell Mol Life Sci. 2008;65:1272-1284.

69. Batelli S, Albani D, Rametta R, et al. DJ-1 modulates alpha-synuclein aggregation state in a cellular model of oxidative stress: relevance for Parkinson's disease and involvement of HSP70. PLoS One. 2008;3:e1884

70. Angeles DC, Gan BH, Onstead L, et al. Mutations in LRRK2 increase phosphorylation of peroxiredoxin 3 exacerbating oxidative stressinduced neuronal death. Hum Mutat. 2011;32:1390-1397. 
71. Sheehan JP, Swerdlow RH, Parker WD, Miller SW, Davis RE, Tuttle JB. Altered calcium homeostasis in cells transformed by mitochondria from individuals with Parkinson's disease. J Neurochem. 1997;68:1221-1233.

72. Trimmer PA, Borland MK, Keeney PM, Bennett JP Jr, Parker WD Jr. Parkinson's disease transgenic mitochondrial cybrids generate Lewy inclusion bodies. J Neurochem. 2004;88:800-812.

73. Esteves AR, Domingues AF, Ferreira IL, et al. Mitochondrial function in Parkinson's disease cybrids containing an nt2 neuron-like nuclear background. Mitochondrion. 2008;8:219-228.

74. Ekstrand MI, Galter D. The MitoPark Mouse - an animal model of Parkinson's disease with impaired respiratory chain function in dopamine neurons. Parkinsonism Relat Disord. 2009;15 Suppl 3:S185-S188.

75. Ekstrand MI, Terzioglu M, Galter D, et al. Progressive parkinsonism in mice with respiratory-chain-deficient dopamine neurons. Proc Natl Acad Sci U S A. 2007;104:1325-1330.

76. Orsucci D, Caldarazzo Ienco E, Mancuso M, Siciliano G. POLG1related and other "mitochondrial Parkinsonisms": an overview. J Mol Neurosci. 2011;44:17-24.

77. Shin JH, Ko HS, Kang H, et al. PARIS (ZNF746) repression of PGC1alpha contributes to neurodegeneration in Parkinson's disease. Cell. 2011;144:689-702.

78. Suen DF, Narendra DP, Tanaka A, Manfredi G, Youle RJ. Parkin overexpression selects against a deleterious mtDNA mutation in heteroplasmic cybrid cells. Proc Natl Acad Sci U S A. 2010;107:11835-11840.

79. De Stefani D, Raffaello A, Teardo E, Szabo I, Rizzuto R. A fortykilodalton protein of the inner membrane is the mitochondrial calcium uniporter. Nature. 2011;476:336-340.

80. Baughman JM, Perocchi F, Girgis HS, et al. Integrative genomics identifies MCU as an essential component of the mitochondrial calcium uniporter. Nature. 2011;476:341-345.

81. Palty R, Silverman WF, Hershfinkel M, et al. NCLX is an essential component of mitochondrial $\mathrm{Na}+\mathrm{Ca} 2+$ exchange. Proc Natl Acad Sci US A. 2010;107:436-441.

82. Rizzuto R, Brini M, Murgia M, Pozzan T. Microdomains with high $\mathrm{Ca} 2+$ close to IP3-sensitive channels that are sensed by neighboring mitochondria. Science. 1993;262:744-747.

83. Rizzuto R, Pinton P, Carrington W, et al. Close contacts with the endoplasmic reticulum as determinants of mitochondrial $\mathrm{Ca} 2+$ responses. Science. 1998;280:1763-1766.

84. de Brito OM, Scorrano L. Mitofusin 2 tethers endoplasmic reticulum to mitochondria. Nature. 2008;456:605-610.

85. Szabadkai G, Bianchi K, Varnai P, et al. Chaperone-mediated coupling of endoplasmic reticulum and mitochondrial Ca2+ channels. J Cell Biol. 2006;175:901-911.

86. Hayashi T, Rizzuto R, Hajnoczky G, Su TP. MAM: more than just a housekeeper. Trends Cell Biol. 2009;19:81-88.

87. Sandebring A, Dehvari N, Perez-Manso M, et al. Parkin deficiency disrupts calcium homeostasis by modulating phospholipase $\mathrm{C}$ signalling. FEBS J. 2009;276:5041-5052.

88. Shtifman A, Zhong N, Lopez JR, Shen J, Xu J. Altered Ca2+ homeostasis in the skeletal muscle of DJ-1 null mice. Neurobiol Aging. 2011; 32:125-132.

89. Furukawa K, Matsuzaki-Kobayashi M, Hasegawa T, et al. Plasma membrane ion permeability induced by mutant alpha-synuclein contributes to the degeneration of neural cells. J Neurochem. 2006;97: 1071-1077.

90. Parihar MS, Parihar A, Fujita M, Hashimoto M, Ghafourifar P. Alphasynuclein overexpression and aggregation exacerbates impairment of mitochondrial functions by augmenting oxidative stress in human neuroblastoma cells. Int J Biochem Cell Biol. 2009;41:2015-2024.

91. Hettiarachchi NT, Parker A, Dallas ML, et al. Alpha-synuclein modulation of $\mathrm{Ca} 2+$ signaling in human neuroblastoma (SH-SY5Y) cells. J Neurochem. 2009;111:1192-1201.

92. Adamczyk A, Strosznajder JB. Alpha-synuclein potentiates $\mathrm{Ca} 2+$ influx through voltage-dependent $\mathrm{Ca} 2+$ channels. Neuroreport. 2006; 17:1883-1886.
93. Calì T, Ottolini D, Negro A, Brini M. Alpha-synuclein controls mitochondrial calcium homeostasis by enhancing endoplasmic reticulum-mitochondria interactions. J Biol Chem. 2012;287: 17914-17929.

94. Cali T, Ottolini D, Negro A, Brini M. Enhanced parkin levels favor ER-mitochondria crosstalk and guarantee $\mathrm{Ca}(2+)$ transfer to sustain cell bioenergetics. Biochim Biophys Acta. 2013;1832:495-508.

95. Ottolini D, Cali T, Negro A, Brini M. The Parkinson disease related protein DJ-1 counteracts mitochondrial impairment induced by the tumor suppressor protein p53 by enhancing endoplasmic reticulummitochondria tethering. Hum Mol Genet. Epub February 14, 2013.

96. Cardenas C, Miller RA, Smith I, et al. Essential regulation of cell bioenergetics by constitutive InsP3 receptor $\mathrm{Ca} 2+$ transfer to mitochondria. Cell. 2010;142:270-283.

97. Marongiu R, Spencer B, Crews L, et al. Mutant Pink1 induces mitochondrial dysfunction in a neuronal cell model of Parkinson's disease by disturbing calcium flux. J Neurochem. 2009;108:1561-1574.

98. Gandhi S, Wood-Kaczmar A, Yao Z, et al. PINK1-associated Parkinson's disease is caused by neuronal vulnerability to calciuminduced cell death. Mol Cell. 2009;33:627-638.

99. Heeman B, Van den Haute C, Aelvoet SA, et al. Depletion of PINK1 affects mitochondrial metabolism, calcium homeostasis and energy maintenance. J Cell Sci. 2011;124:1115-1125.

100. Cherra SJ 3rd, Steer E, Gusdon AM, Kiselyov K, Chu CT. Mutant LRRK2 elicits calcium imbalance and depletion of dendritic mitochondria in neurons. Am J Pathol. 2013;182:474-484.

101. Avery MA, Rooney TM, Pandya JD, et al. WldS prevents axon degeneration through increased mitochondrial flux and enhanced mitochondrial Ca2+ buffering. Curr Biol. 2012;22:596-600.

102. Kitay BM, McCormack R, Wang Y, Tsoulfas P, Zhai RG. Mislocalization of neuronal mitochondria reveals regulation of Wallerian degeneration and NMNAT/WLDS-mediated axon protection independent of axonal mitochondria. Hum Mol Genet. Epub January 24, 2013.

103. Ocampo A, Liu J, Barrientos A. NAD+ salvage pathway proteins suppress proteotoxicity in yeast models of neurodegeneration by promoting the clearance of misfolded/oligomerized proteins. Hum Mol Genet. Epub January 30, 2013.

104. Antenor-Dorsey JA, O’Malley KL. WldS but not Nmnat1 protects dopaminergic neurites from MPP+ neurotoxicity. Mol Neurodegener. 2012;7:5.

105. Westermann B. Mitochondrial fusion and fission in cell life and death. Nat Rev Mol Cell Biol. 2010;11:872-884.

106. Smirnova E, Griparic L, Shurland DL, van der Bliek AM. Dynaminrelated protein Drp1 is required for mitochondrial division in mammalian cells. Mol Biol Cell. 2001;12:2245-2256.

107. Cereghetti GM, Stangherlin A, Martins de Brito O, et al. Dephosphorylation by calcineurin regulates translocation of Drp1 to mitochondria. Proc Natl Acad Sci U S A. 2008;105:15803-15808.

108. Mears JA, Lackner LL, Fang S, Ingerman E, Nunnari J, Hinshaw JE. Conformational changes in Dnm1 support a contractile mechanism for mitochondrial fission. Nat Struct Mol Biol. 2011;18:20-26.

109. Mozdy AD, McCaffery JM, Shaw JM. Dnm1p GTPase-mediated mitochondrial fission is a multi-step process requiring the novel integral membrane component Fis1p. J Cell Biol. 2000;151:367-380.

110. Lee YJ, Jeong SY, Karbowski M, Smith CL, Youle RJ. Roles of the mammalian mitochondrial fission and fusion mediators Fis1, Drp1, and Opa1 in apoptosis. Mol Biol Cell. 2004;15:5001-5011.

111. Otera H, Wang C, Cleland MM, et al. Mff is an essential factor for mitochondrial recruitment of Drp1 during mitochondrial fission in mammalian cells. J Cell Biol. 2010;191:1141-1158.

112. Friedman JR, Lackner LL, West M, DiBenedetto JR, Nunnari J, Voeltz GK. ER tubules mark sites of mitochondrial division. Science. 2011;334:358-362.

113. Cipolat S, Martins de Brito O, Dal Zilio B, Scorrano L. OPA1 requires mitofusin 1 to promote mitochondrial fusion. Proc Natl Acad Sci USA. 2004;101:15927-15932. 
114. Corrado M, Scorrano L, Campello S. Mitochondrial dynamics in cancer and neurodegenerative and neuroinflammatory diseases. Int $J$ Cell Biol. 2012;2012:729290.

115. Wang X, Petrie TG, Liu Y, Liu J, Fujioka H, Zhu X. Parkinson's disease-associated DJ-1 mutations impair mitochondrial dynamics and cause mitochondrial dysfunction. J Neurochem. 2012;121: 830-839.

116. Wang H, Song P, Du L, et al. Parkin ubiquitinates Drp1 for proteasome-dependent degradation: implication of dysregulated mitochondrial dynamics in Parkinson disease. J Biol Chem. 2011;286: 11649-11658.

117. Wang X, Yan MH, Fujioka H, et al. LRRK2 regulates mitochondrial dynamics and function through direct interaction with DLP1. Hum Mol Genet. 2012;21:1931-1944.

118. Zhang Q, Wu J, Wu R, et al. DJ-1 promotes the proteasomal degradation of Fis 1: implications of DJ-1 in neuronal protection. Biochem J. 2012;447:261-269.

119. Santos D, Cardoso SM. Mitochondrial dynamics and neuronal fate in Parkinson's disease. Mitochondrion. 2012;12:428-437.

120. Pilsl A, Winklhofer KF. Parkin, PINK1 and mitochondrial integrity: emerging concepts of mitochondrial dysfunction in Parkinson's disease. Acta Neuropathol. 2012;123:173-188.

121. Whitworth AJ, Pallanck LJ. The PINK1/Parkin pathway: a mitochondrial quality control system? J Bioenerg Biomem. 2009;41:499-503.

122. Krebiehl G, Ruckerbauer S, Burbulla LF, et al. Reduced basal autophagy and impaired mitochondrial dynamics due to loss of Parkinson's disease-associated protein DJ-1. PLoS One. 2010;5:e9367.

123. Irrcher I, Aleyasin H, Seifert EL, et al. Loss of the Parkinson's diseaselinked gene DJ-1 perturbs mitochondrial dynamics. Hum Mol Genet. 2010;19:3734-3746

124. Thomas KJ, McCoy MK, Blackinton J, et al. DJ-1 acts in parallel to the PINK1/parkin pathway to control mitochondrial function and autophagy. Hum Mol Genet. 2011;20:40-50.

125. Zhu M, Li W, Lu C. Role of alpha-synuclein protein levels in mitochondrial morphology and cell survival in cell lines. PLoS One. 2012;7: e36377.

126. Rhinn H, Qiang L, Yamashita T, et al. Alternative $\alpha$-synuclein transcript usage as a convergent mechanism in Parkinson's disease pathology. Nat Commun. 2012;3:1084

127. Niu J, Yu M, Wang C, Xu Z. Leucine-rich repeat kinase 2 disturbs mitochondrial dynamics via dynamin-like protein. JNeurochem. 2012; 122:650-658.

128. Weihofen A, Thomas KJ, Ostaszewski BL, Cookson MR, Selkoe DJ. Pink1 forms a multiprotein complex with Miro and Milton, linking Pink1 function to mitochondrial trafficking. Biochemistry. 2009;48: 2045-2052.

129. Wang X, Winter D, Ashrafi G, et al. PINK1 and Parkin target Miro for phosphorylation and degradation to arrest mitochondrial motility. Cell. 2011;147:893-906.

130. Liu S, Sawada T, Lee S, et al. Parkinson's disease-associated kinase PINK1 regulates Miro protein level and axonal transport of mitochondria. PLoS Genet. 2012;8:e1002537.
131. Larsen NJ, Ambrosi G, Mullett SJ, Berman SB, Hinkle DA. DJ-1 knock-down impairs astrocyte mitochondrial function. Neuroscience. 2011;196:251-264.

132. Xie W, Chung KK. Alpha-synuclein impairs normal dynamics of mitochondria in cell and animal models of Parkinson's disease. J Neurochem. 2012;122:404-414.

133. de Vries RL, Przedborski S. Mitophagy and Parkinson's disease: be eaten to stay healthy. Mol Cell Neurosci. Epub August 2, 2012.

134. Imai Y, Lu B. Mitochondrial dynamics and mitophagy in Parkinson's disease: disordered cellular power plant becomes a big deal in a major movement disorder. Curr Opin Neurobiol. 2011;21:935-941.

135. Tanaka A, Cleland MM, Xu S, et al. Proteasome and p97 mediate mitophagy and degradation of mitofusins induced by Parkin. $J$ Cell Biol. 2010;191:1367-1380.

136. Ziviani E, Tao RN, Whitworth AJ. Drosophila parkin requires PINK1 for mitochondrial translocation and ubiquitinates mitofusin. Proc Natl Acad Sci U S A. 2010;107:5018-5023.

137. Geisler S, Holmstrom KM, Skujat D, et al. PINK1/Parkin-mediated mitophagy is dependent on VDAC1 and p62/SQSTM1. Nat Cell Biol. 2010;12:119-131.

138. Chen D, Gao F, Li B, et al. Parkin mono-ubiquitinates Bcl-2 and regulates autophagy. J Biol Chem. 2010;285:38214-38223.

139. Lee JY, Nagano Y, Taylor JP, Lim KL, Yao TP. Disease-causing mutations in parkin impair mitochondrial ubiquitination, aggregation, and HDAC6-dependent mitophagy. J Cell Biol. 2010;189:671-679.

140. Narendra DP, Jin SM, Tanaka A, et al. PINK1 is selectively stabilized on impaired mitochondria to activate Parkin. PLoS Biol. 2010;8:e1000298.

141. Okatsu K, Oka T, Iguchi M, et al. PINK1 autophosphorylation upon membrane potential dissipation is essential for Parkin recruitment to damaged mitochondria. Nat Commun. 2012;3:1016.

142. Chen Y, Dorn G II. PINK1-phosphorylated mitofusin 2 is a parkin receptor for culling damaged mitochondria. Science. 2013;340:471-475.

143. Cuervo AM, Stefanis L, Fredenburg R, Lansbury PT, Sulzer D. Impaired degradation of mutant alpha-synuclein by chaperonemediated autophagy. Science. 2004;305:1292-1295.

144. Sampaio-Marques B, Felgueiras C, Silva A, et al. SNCA (alphasynuclein)-induced toxicity in yeast cells is dependent on sirtuin 2 (Sir2)-mediated mitophagy. Autophagy. 2012;8:1494-1509.

145. Gao H, Yang W, Qi Z, et al. DJ-1 protects dopaminergic neurons against rotenone-induced apoptosis by enhancing ERK-dependent mitophagy. J Mol Biol. 2012;423:232-248.

146. Joselin AP, Hewitt SJ, Callaghan SM, et al. ROS-dependent regulation of Parkin and DJ-1 localization during oxidative stress in neurons. Hum Mol Genet. 2012;21:4888-4903.

147. Manzoni C. LRRK2 and autophagy: a common pathway for disease. Biochem Soc Trans. 2012;40:1147-1151.

148. Moreira PI, Zhu X, Wang X, et al. Mitochondria: a therapeutic target in neurodegeneration. Biochim Biophys Acta. 2010;1802:212-220.
Research and Reports in Biochemistry

\section{Publish your work in this journal}

Research and Reports in Biochemistry is an international, peer-reviewed, open access journal publishing original research, reports, reviews and commentaries on all areas of biochemistry. The manuscript management system is completely online and includes a very quick and fair
Dovepress

peer-review system. Visit http://www.dovepress.com/testimonials.php to read real quotes from published authors. 